\title{
Impact of COVID-19 on Clinical Biochemistry: Indian Scenario
}

\author{
Prasenjit Mitra $^{1} \cdot$ Sanjeev Misra ${ }^{2} \cdot$ Praveen Sharma $^{3}$
}

Published online: 6 September 2021

(C) Association of Clinical Biochemists of India 2021

The Indian Government reported the country's first coronavirus disease 2019 (COVID-19) case on January 30, 2020. The patient was a University student who returned to Kerala from Wuhan Province, China, where the first case of COVID-19 was reported in December 2020. At that point, nobody probably thought that this disease would change the way we live, socialize and practise our professions. The disease spread in such a large proportion distressing numerous countries that changed our perspectives towards patient healthcare. COVID-19 was declared a global pandemic by WHO on March 11, 2020 [1]. The next one and a half years have been the most challenging times for the entire medical community. As of August 31, 2021, around 218,561,740 diagnosed cases of COVID-19 with 4,534,055 deaths and 195,396,786 recoveries have been reported. The United States of America (USA), India, Brazil, Russia, France have been the most affected countries. In India, the total cases of COVID-19 have been $32,810,892$, with 439,054 deaths [2].

To stop the spread of COVID-19, India announced a nationwide lockdown which started on March 24, 2020, and continued for 21 days. When compared to measures implemented by other countries, the Indian lockdown was the strongest of all with many restrictions, including the complete closure of educational institutions, travel bans,

Praveen Sharma

praveensharma55@gmail.com

1 Department of Biochemistry, Postgraduate Institute of Medical Education and Research, Chandigarh, India

2 Department of Surgical Oncology, All India Institute of Medical Sciences, Jodhpur, India

3 Department of Biochemistry, All India Institute of Medical Sciences, Jodhpur, India border closure, and many other. As per the "Oxford COVID-19 Government Response Tracker", designed to systematically record government responses worldwide and aggregate the scores into a standard 'Stringency Index', India scored a perfect 100, meaning that the Government had implemented all necessary measures to contain the virus spread. The lockdown was successful in retarding the spread of the infection. Before lockdown, the doubling rate, every 3 days, significantly slowed down to eight days after completing the first phase [3].

The first wave of COVID-19 was followed by a second wave, which had devastating consequences in the form of exponentially increasing cases, decreased supplies of necessary treatments, and notably higher numbers of deaths in the younger population. The entire nation went through a challenging time managing the second wave, as several new things happened that were not predominant in the first wave. Identification of several double and triple mutant strains throughout the country with altered transmissibility and increased pathogenicity than initial strains indicated that the virus evolved with time [4]. Another alarming event during the second wave was the high incidence of mucormycosis (black fungus) in COVID-19 patients, individuals with co-morbid conditions like Diabetes, and in subjects under-recovery. Unwarranted use of steroids as a part of COVID-19 management strategy and virus-induced immunosuppression led to this additional problem. Now that the second wave is over, it becomes essential to explore why it was much more severe than the first, as this may lead to the identification of newer diagnostic areas to target so that a novel strategy may be formulated to combat the third wave in the future [5].

From detecting the pathogen via reverse transcriptasepolymerase chain reaction (RT-PCR) to quantifying the antibody responses towards COVID-19, laboratory 
professionals played a significant role during this pandemic. In a developing country like India, where $>80 \%$ of the clinical biochemistry laboratories are small and without adequate physical and human resources, COVID-19 posed a significant threat by presenting several challenges. These included inadequate availability of human resources, transportation issues, lack of personal protective equipment (PPE), delayed delivery and sometimes unavailability of essential supplies, deteriorating revenue targets, health-related problems of the employees, and most importantly, a growing fear and anxiety amongst the frontline staff. Despite all these challenges, the laboratory professionals provided the best possible diagnostic support to the clinicians in this public health crisis [3].

An unprecedented shift in healthcare resources was observed with the outbreak of COVID-19. Though there was an increase in hospitalization and patients with acute illness, various surgical procedures and outpatient appointments and delays in non-urgent health issues were also noted. These issues led to a significant drop in the laboratory test group and severely impacted clinical laboratory testing. Furthermore, a decrease in the test requisition due to COVID-19 significantly altered the requirement of laboratory personnel, reagents, and analyzers, causing a substantial financial burden and revenue generation targets to remain unmet [6].

Following the various national and international guidelines, a limited number of tests, including total blood count, Ferritin, CRP, blood gas, and D-dimer, were recommended for COVID-19 patients. Further, as and when required, depending on the severity, procalcitonin, troponin, prothrombin time, and routine biochemical tests were also analyzed [7]. Despite a reduction in routine tests, more than usual demands have dramatically crashed the reagent management in clinical laboratories. While, due to increased demand for a particular test, the laboratories had to borrow kits from other sources, some biochemistry tests had to be discontinued, as there were shipment delays from manufacturers due to the lockdown imposed by Government. Furthermore, to meet the rapid surge in the inpatient admission of COVID-19 patients and increasing demand for POCT, urgent procurement and installation of POCTs were done in various hospital settings. The non-availability of the test kits and consumables further led to disruption in the scheduled method validation and verification activities, which are among the prime requirements for the quality assurance and accreditation processes [3].

Several arrangements were followed to reduce the potential risk of staff exposure. The clinical laboratory professionals were divided into teams to minimize contact, wherein the other team members did not meet or work in the overlapping shifts. In some scenarios, it was challenging due to inadequate staff, leading to longer working hours. Beyond the workload experienced by the laboratory professionals, the lockdown had severely affected the workflow in the clinical laboratories. Due to the closure of public transport, there was a problem in the commutation of the laboratory professionals. Not only this, there was a continuous threat of losing jobs, salary cuts, postponed appraisals among the lab professionals. It further aggravated the mental health of the employees who were already under threat of communicating the disease to their family members by working in such a contagious environment [8].

Amid the speculations for contracting the third wave of COVID-19 in India, it becomes inevitable to stick to various lessons, adaptation, experiences the laboratories have gained during the past exposure to this pandemic. With a proactive approach, the laboratory professionals should be ready with an acceptable alternate plan for better health outcomes. The varying trend of laboratory tests and inadequate supply of reagents and consumables thus ensue an urgent need for optimal inventory management during this era. Earlier adaptation to the changes will help create a better, safer, and workable environment for all.

\section{References}

1. Mitra P, Misra S, Sharma P. COVID-19 Pandemic in India: What lies ahead. Ind J Clin Biochem. 2020;35(3):257-9. https://doi.org/ 10.1007/S12291-020-00886-6.

2. Worldometers.info. 2021. COVID Live Update: $218,567,248$ Cases and 4,534,150 Deaths from the Coronavirus-Worldometer. https://www.worldometers.info/coronavirus/. Accessed 1 Sep 2021

3. Mitra P, Misra S, Sharma P. One year of COVID-19: the "new normal." Ind J Clin Biochem. 2021;36(1):1-2.

4. Choudhary S, Sreenivasulu K, Mitra P, Misra S, Sharma P. Role of genetic variants and gene expression in the susceptibility and severity of COVID-19. Ann Lab Med. 2021;41(2):129-38. https:// doi.org/10.3343/alm.2021.41.2.129.

5. Asrani P, Eapen MS, Hassan MI, Sohal SS. Implications of the second wave of COVID-19 in India. Lancet Respir Med. 2021;9:e93-4.

6. Durant TJS, Peaper DR, Ferguson D, Schulz WL. Impact of COVID-19 Pandemic on laboratory utilization. J Appl Lab Med. 2020;5(6):1194-205.

7. Lingeswaran M, Goyal T, Ghosh R, Suri S, Mitra P, Misra S, et al. Inflammation, immunity and immunogenetics in COVID-19: a narrative review. Ind J Clin Biochem. 2020;35(3):260-73. https:// doi.org/10.1007/S12291-020-00897-3.

8. Kabanova A, Gavriilaki E, Pelzer BW, Brunetti L, Maiques-Diaz A. Effect of the COVID-19 Pandemic on laboratory and clinical research: a testimony and a call to action from researchers. HemaSphere. 2020;4(6):e499.

Publisher's Note Springer Nature remains neutral with regard to jurisdictional claims in published maps and institutional affiliations. 